\title{
Serum Progesterone and Serial Beta-hcG Levels in Predicting the Outcome of Early Pregnancies With Doubtful Viability: A Prospective Observational Research
}

Mustafa YILDIZ ( $\nabla$ dr.mustafayldz@gmail.com )

Private Derman Hospital

Esra Nur CICEK

Bartin State Hospital

Gokhan DEMIRAYAK

Istanbul Bakirkoy Dr. Sadi Konuk Training and Research Hospital

Orhan SAHIN

Istanbul Prof.Dr. Cemil Tascioglu Okmeydani City Hospital

Yagmur OLMEZ

Istanbul Prof.Dr. Cemil Tascioglu Okmeydani City Hospital

Veli MIHMANLI

Istanbul Prof.Dr. Cemil Tascioglu Okmeydani City Hospital

\section{Research Article}

Keywords: Early pregnancy loss, viability, serial $\beta$-hCG, progesterone

Posted Date: September 16th, 2021

DOl: https://doi.org/10.21203/rs.3.rs-842511/v1

License: (c) (i) This work is licensed under a Creative Commons Attribution 4.0 International License.

Read Full License 


\section{Abstract}

BACKGROUND: To evaluate the doubling rate of maternal serum serial $\beta$-hCG and a single initial serum progesterone level to predict fetal viability before ultrasonography in women diagnosed with intrauterine pregnancy of undetectable viability.

METHODS: 336 pregnant women who applied to the outpatient clinic at Istanbul Okmeydanı City Hospital between March and December 2018 were evaluated on a "Prospective observational" basis. 236 women were excluded from the study for various reasons. The study was completed with 100 pregnant women diagnosed with intrauterine pregnancy (IUP) involving suspected fetal viability by transvaginal ultrasonography only, who met the inclusion criteria with CRL: $<7 \mathrm{~mm}$ and mean gestational sac diameter: < 25mm. Serum $\beta$-hCG and progesterone were taken at the first admission. After 48 hours, control serum $\beta$-hCG was taken and the increase rates were calculated. Early pregnancy loss was diagnosed by (transvaginal) TV-USG performed after the days 7, 11 and 14. Patients were divided into two groups as fetal heart rate (FHR) positive (viable) and FHR negative (early pregnancy loss). Pregnancy results were compared with $\beta$-hCG increase rates and progesterone value. SPSS 22.0 software was used for statistical analysis and $P<0.05$ was accepted as statistically significant.

RESULTS: No statistically significant result was obtained between the viable and early pregnancy loss $(\mathrm{FHR}+/-)$ groups in terms of maternal age, previous pregnancy anamnesis, nationality, presenting symptoms, or ultrasound findings. According to the last menstrual period, the mean gestational age was $45.1 \pm 14$ days in the viable (FHR+) group and $51.3 \pm 14$ days in the Early pregnancy loss (FHR-) group, and this difference was found to be statistically significant. Estimation modality was developed in terms of viability with the serum progesterone values and increase rates of $\beta$-hCG. The study, which was conducted with a confidence interval of $95 \%$, found the viability rate to be $70 \%$ with a $\beta$-hCG increase rate of $31 \%, 80 \%$ in the case of an increase by $49 \%, 90 \%$ in the case of an increase by $73 \%, 95 \%$ in the case of an increase by $97 \%$, and $100 \%$ in the case of an increase by $181 \%$. For progesterone, when the value was $5.9 \mathrm{ng} / \mathrm{ml}$, the viability rate was $49 \%$, and it was $69 \%$ at $10.5 \mathrm{ng} / \mathrm{ml}, 80 \%$ at $13.4 \mathrm{ng} / \mathrm{ml}, 90 \%$ at 18.0 $\mathrm{ng} / \mathrm{ml}, 95 \%$ at $21.7 \mathrm{ng} / \mathrm{ml}, 99 \%$ at $29.3 \mathrm{ng} / \mathrm{ml}$, and $100 \%$ at $37.5 \mathrm{ng} / \mathrm{ml}$ and above. In conclusion, the significant efficacy values of $\beta$-hCG increase and first progesterone level in predicting viability were found to be ROC AUC: [0.748 (0.621-0.874)] and ROC AUC: [0.796 (0.685-0.907)], respectively.

CONCLUSION: Either Serial $\beta$-hCG ratio or serum progesterone level can be used alone to predict pregnancy outcome in early pregnancy. With dissemination of similar studies, estimation modalities can be improved and TV-USG examinations can help shortening the waiting time for results to reduce anxiety of families, hospital admissions and health expenses.

\section{Introduction:}

Early pregnancy loss is a frequently encountered problem in the reproductive period, which is the longest period of female life, and it is reported as the most common complication of pregnancy. It occurs in 
about $10-15 \%$ of all clinically recognized pregnancies and affects one in every three women (1). Approximately $80 \%$ of all pregnancy loss cases occur in the first quarter (2).

Bleeding and pain are the most common reasons why women receive medical advice during their early pregnancy. This is an important cause of anxiety for women. Detection of intrauterine pregnancy (IUP) by Transvaginal Ultrasonography (TV-USG) following a positive $\beta$-hCG excludes ectopic pregnancy except heterotopic pregnancy, but especially during the follow-up of Pregnancy of Unknown Locations (PUL), pregnancy viability cannot be determined at the first visit when IUP is detected (3). When an IUP is detected, the second most important step is to determine viability. These one- or two-week follow-up visits may cause long-term anxiety for women, and these visits increase the cost and workload in the related healthcare facilities (4).

Serial measurements of serum hCG, the increase percentages, and serum progesterone measurement used for the same purpose as serum hCG in early pregnancies are highly important in differentiation of normal and abnormal pregnancies as well as in prediction of fetal viability. However, both involve significant differences in the literature (5). No cut-off value or predictive power of any of these markers could be determined for the definitive diagnosis of viability in IUPs.

The aim of this study is to compare maternal serum series $\beta-h C G$ and a single serum Progesterone level with TV-USG for predicting fetal viability in women with intrauterine pregnancy and create a prediction modality using only serum markers.

\section{Materials And Methods:}

Our study was carried out in the Obstetrics and Gynecology Clinic of Istanbul Okmeydani City Hospital on a single-center "Prospective observational" basis between March 2018 and December 2018. The study was commenced after obtaining the approval of the Ethics Committee for Clinical Research of Istanbul Okmeydani City Hospital under the protocol number 841 on 13/03/2018. The participants were informed verbally and in writing, and their written consents were obtained.

As a result of the Power analysis performed using the $\mathrm{G}$ PPower software, taking the effect size for hCG 'H48' and Progesterone ' $P$ ' parameters as 0.1806 and SD: 17538, the minimum number of samples determined for Power:0.80 anda:0.05 was determined as n:100 for each group. Inclusion criteria were defined as consent for participating in the study, being older than 18 years of age, diagnosis of intrauterine pregnancy by TV-USG, and early pregnancy with no fetal heartbeat detected / suspected (CRL $<7 \mathrm{~mm}, \mathrm{GS}<25 \mathrm{~mm}$ ). Our study includes 336 pregnant women who applied to our outpatient clinic and accepted to participate in the study. 156 pregnant women who did not meet at least one of the inclusion criteria, and 28 pregnant women did not want to continue in the study, despite they initially agreed to participate in it, and 20 pregnant women having other reasons were excluded from the study. During the follow-ups, 32 pregnant women could not be reached and they were also excluded from the study. So, the study was completed with 100 pregnant women (Table-1). All women were evaluated by experienced obstetricians using ultrasound devices equipped with 6-12 $\mathrm{MHz}$ transvaginal transducer 
and B-mode imaging. Pregnancy of Uncertain Viability (PUV) criteria in patients with intrauterine pregnancy confirmed by TV-USG were taken as no heart rate below $\mathrm{CRL}<7 \mathrm{~mm}$, and no embryo below $\mathrm{GS}<25 \mathrm{~mm}$ (mean of three orthogonal measurements: $\mathrm{mGSD}$ ). Anamnesis information such as maternal age, previous pregnancy anamnesis, nationality, admission symptoms, and ultrasound findings of the patients were noted at the first admission. Viability determination was performed by TV-USG on the days 7,11 , and 14, respectively. Pregnant women with fetal heartbeat detected, or diagnosed with fetal viability (Viable: FHR: + ) were not invited to further controls. Pregnant women with undetectable fetal heart rate were held until the day 14 at the latest, and if no fetal heart rate could be detected still, they were diagnosed with "Early pregnancy loss" (Non-viable: FHR: -).

Serum $\beta$-hCG and progesterone were taken at the first admission. After 48 hours, control serum $\beta$-hCG was taken and the increase rates were calculated. Serum plasmic $h C G$ and progesterone levels were measured with Roche hCG and Progesterone II Electrochemiluminescence Immunassay (ECLIA) in Roche Cobas e411 and e602 analyzers, respectively.

As a result, patients were divided into two groups as fetal heart rate positive group (FHR: + ) and early pregnancy loss group (FHR: -). These results were interpreted with the percentage of serum $\beta$-hCG increase and Progesterone value at initial admission in the light of the literature. These two parameters were evaluated by means of Mann-Whitney u test and Wilcoxon test. However, z-scores and percentiles adjusted according to gestational week were used to calculate both $\beta$-hCG and serum progesterone levels. Thus, it was considered that the first progesterone level was not constant and $\beta$-hCG levels would decrease over time. Receiver Operating Characteristic (ROC) curve was used for the estimations, effect level and cutoff values of numerical parameters. SPSS 22.0 (Chicago, IL, USA) was used for statistical analysis.

\section{Results:}

Our study includes 336 pregnant women who applied to our outpatient clinic and accepted to participate in the study. 156 pregnant women who did not meet at least one of the inclusion criteria, and 28 pregnant women did not want to continue in the study, despite they initially agreed to participate in it, and 20 pregnant women having other reasons were excluded from the study. During the follow-ups, 32 pregnant women could not be reached and they were also excluded from the study. So, the study was completed with 100 pregnant women (Table-1).

Table-1: Consort Diagram 


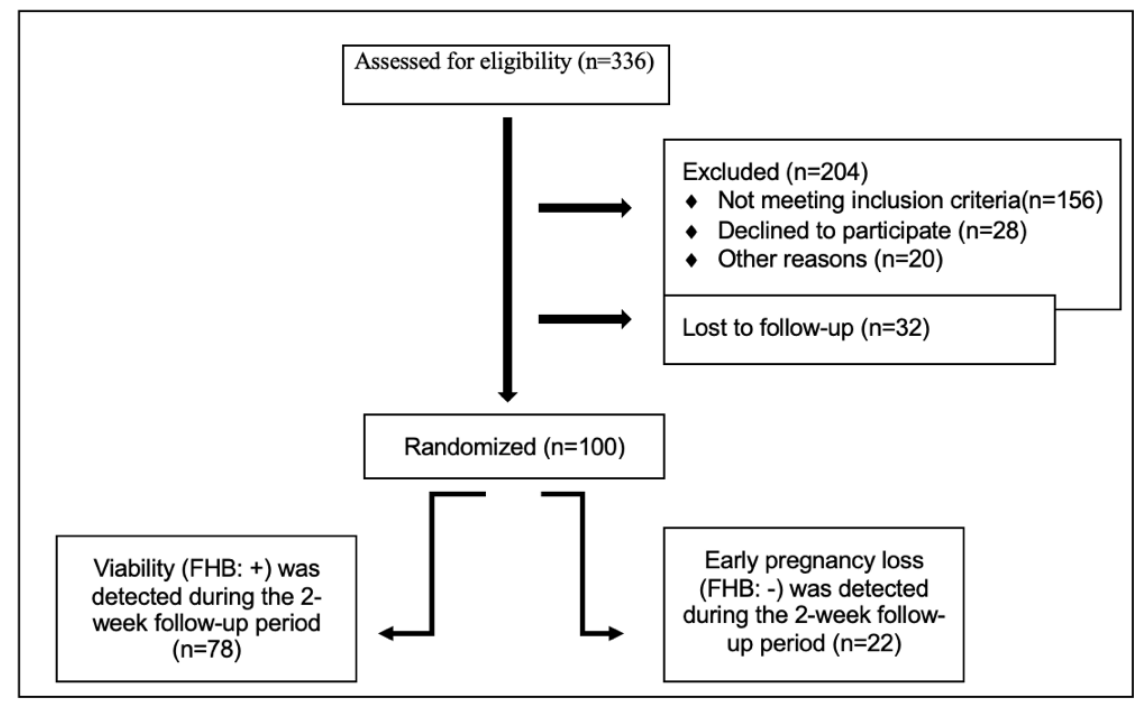

The Table-2 shows the age, pregnancy history, LMP (Last menstrual period), nationality, presenting symptom, previous mode of pregnancy, viability, $\beta$-hCG values (control at first admission and at hour 48) and percentage of increase, serum progesterone value at first admission, TV-USG findings and measurements of the patients participating in the study.

Table-2: Anamnesis, Ultrasound and Laboratory findings of the patients participating in the study

\begin{tabular}{|c|c|c|c|c|c|}
\hline & & Min-Max & Median & \multicolumn{2}{|c|}{ Mean \pm S.d. $/ n-\%$} \\
\hline \multicolumn{2}{|l|}{ Age } & $18,0-42,0$ & 29,0 & 28,8 & $\pm 5,7$ \\
\hline \multicolumn{2}{|l|}{ Gravida } & $1,0-8,0$ & 2,0 & 2,6 & $\pm 1,3$ \\
\hline \multicolumn{2}{|l|}{ Parity } & $0,0-3,0$ & 1,0 & 1,0 & $\pm 0,9$ \\
\hline \multicolumn{2}{|l|}{ Abortion } & $0,0-5,0$ & 0,0 & 0,5 & $\pm 0,8$ \\
\hline \multicolumn{2}{|l|}{ Curettage } & $0,0-2,0$ & 0,0 & 0,1 & $\pm 0,3$ \\
\hline \multirow{2}{*}{ Ektopic Pregnancy } & $(-)$ & & & 98 & $98,0 \%$ \\
\hline & $(+)$ & & & 2 & $2,0 \%$ \\
\hline \multicolumn{2}{|c|}{ Last Menstrual Period (Day) } & $21,0-116,0$ & 44,0 & 46,4 & $\pm 14,1$ \\
\hline \multirow{2}{*}{ Nationality } & Turkish & & & 97 & $97,0 \%$ \\
\hline & Foreign & & & 3 & $3,0 \%$ \\
\hline \multicolumn{6}{|l|}{ Symptom } \\
\hline \multicolumn{2}{|l|}{ No } & & & 43 & $43,0 \%$ \\
\hline \multicolumn{2}{|c|}{ Mens. delay / Pregnancy suspicon } & & & 19 & $19,0 \%$ \\
\hline \multicolumn{2}{|l|}{ Bleeding/Spotting } & & & 21 & $21,0 \%$ \\
\hline \multicolumn{2}{|l|}{ Waist / Groin pain } & & & 15 & $15,0 \%$ \\
\hline \multicolumn{2}{|l|}{ Others } & & & 2 & $2,0 \%$ \\
\hline \multirow{4}{*}{ Previously Pregnancy } & Nulliparous & & & 32 & $32,0 \%$ \\
\hline & Vag. Delivery & & & 34 & $34,0 \%$ \\
\hline & Cesarean Sect. & & & 31 & $31,0 \%$ \\
\hline & Vag. Del. +C/S & & & 3 & $3,0 \%$ \\
\hline \multirow{2}{*}{ Viability } & Early Pregnancy & (FHB: -) & & 22 & $22,0 \%$ \\
\hline & Detected Viabilit & $\mathrm{HB}:+)$ & & 78 & $78,0 \%$ \\
\hline \multicolumn{6}{|l|}{ BHCG (All cases) } \\
\hline \multicolumn{2}{|l|}{ First Examination } & $302-200727$ & 18430 & 25880 & \pm 30951 \\
\hline \multicolumn{2}{|l|}{ Control } & $848-235654$ & 28817 & 35962 & \pm 38268 \\
\hline \multicolumn{2}{|l|}{ Percentage İncrease (\%) } & $0,8-181,3$ & 52,5 & 54,3 & $\pm 36,2$ \\
\hline \multicolumn{6}{|l|}{ In-Group Change (p) } \\
\hline \multicolumn{2}{|l|}{ Progesteron } & $3,3-50,0$ & 13,7 & 15,5 & $\pm 8,3$ \\
\hline \multirow{2}{*}{ USG } & mGSD (No CRL) & & & 81 & $81,0 \%$ \\
\hline & CRL detected & & & 19 & $19,0 \%$ \\
\hline
\end{tabular}


The mean age of all patients was $28.8 \pm 5.7$, the mean number of Gravida was $2.6 \pm 1.3$ and the mean Parity was $1.0 \pm 0.9$. The most common presenting symptom was "Bleeding/Maculation" with $21 \%$ (n:21). $32 \%$ (n:32) of the women were nulliparous and $68 \%$ (n:68) were multiparous. The mean increase in beta HCG was $54.3 \pm 36.2 \%$ and the mean Progesterone value at first admission was $15.5 \pm 8.3$. Fetal heart rate was positive (viability + ) in $78 \%$ of women (n:78) and negative (early pregnancy loss) in $22 \%$ (n:22).

Table-3: Comparison of demographic characteristics and symptoms of patients with fetal heart rate positive (Viable) or negative (Early pregnancy loss)

\begin{tabular}{|c|c|c|c|c|c|c|c|c|}
\hline & & \multicolumn{3}{|c|}{ Early Pregnancy Loss (FHB: -) } & \multicolumn{3}{|c|}{ Detected Viability (FHB: +) } & \multirow{2}{*}{ P } \\
\hline & & Mean : & S.d. $/ \mathrm{n}-\%$ & Median & Mean : & S.d./n-\% & Median & \\
\hline \multicolumn{2}{|l|}{$\overline{\text { Age }}$} & 29,7 & $\pm 5,0$ & 30,0 & 28,5 & $\pm 6,0$ & 28,0 & $0,408^{t}$ \\
\hline \multicolumn{2}{|l|}{ Gravida } & 3,1 & $\pm 1,7$ & 3,0 & 2,4 & $\pm 1,2$ & 2,0 & $0,144 \mathrm{~m}$ \\
\hline \multicolumn{2}{|l|}{ Parity } & 1,2 & $\pm 1,1$ & 1,0 & 1,0 & $\pm 0,9$ & 1,0 & $0,610 \mathrm{~m}$ \\
\hline \multicolumn{2}{|l|}{ Abortion } & 0,8 & $\pm 1,0$ & 0,0 & 0,4 & $\pm 0,7$ & 0,0 & $0,079 \mathrm{~m}$ \\
\hline \multicolumn{2}{|l|}{ Curettage } & 0,1 & $\pm 0,4$ & 0,0 & 0,1 & $\pm 0,3$ & 0,0 & $0,864 \mathrm{~m}$ \\
\hline \multicolumn{2}{|c|}{ Last Menstrual Period (Day) } & 51,3 & $\pm 14,0$ & 49,0 & 45,1 & $\pm 14,0$ & 44,0 & $0,040^{\mathrm{m}}$ \\
\hline \multirow{2}{*}{ Nationality } & Turkish & 21 & $95,5 \%$ & & 76 & $97,4 \%$ & & \multirow{2}{*}{$0,530^{x^{2}}$} \\
\hline & Foreign & 1 & $4,5 \%$ & & 2 & $2,6 \%$ & & \\
\hline \multicolumn{9}{|l|}{ Symptom } \\
\hline \multicolumn{2}{|l|}{ No } & 10 & $45,5 \%$ & & 33 & $42,3 \%$ & & \multirow{5}{*}{$0,9844^{x^{2}}$} \\
\hline \multicolumn{2}{|c|}{ Mens. delay / Pregnancy } & 2 & $9,1 \%$ & & 17 & $21,8 \%$ & & \\
\hline \multicolumn{2}{|c|}{ Bleeding / Spotting } & 7 & $31,8 \%$ & & 14 & $17,9 \%$ & & \\
\hline \multicolumn{2}{|c|}{ Waist / Groin pain } & 3 & $13,6 \%$ & & 12 & $15,4 \%$ & & \\
\hline \multicolumn{2}{|l|}{ Others } & 0 & $0,0 \%$ & & 2 & $2,6 \%$ & & \\
\hline
\end{tabular}

${ }^{\mathrm{t}} \mathrm{t}$ test $/{ }^{\mathrm{m}}$ Mann-whitney u test $/{ }^{\mathrm{x}^{2}} \mathrm{Ki}$-kare test (Fischer test)

No significant statistical results were obtained in the comparison of maternal age, gravida number, parity number, nationality and presenting symptoms between the fetal heartbeat negative and positive groups. The difference between the gestational ages was found to be statistically significant only between the two groups according to their last menstrual periods (P: 0.040).

Table 4 : Comparison of serial $\beta-h C G$ and percentage of increase between FHR +/- (Viable / Non-viable: Early pregnancy loss) groups

\begin{tabular}{|c|c|c|c|}
\hline & Early Pregnancy Loss (FHB: -) & Detected Viability (FHB: + ) & \\
\hline & Mean \pm S.d. & Mean \pm S.d. & $p$ \\
\hline \multicolumn{4}{|l|}{ BHCG } \\
\hline First Examination & $16874 \pm 16029$ & $28420 \pm 33644$ & $0,090^{m}$ \\
\hline Control & $20031 \pm 16602$ & $40455 \pm 41409$ & $0,012 \mathrm{~m}$ \\
\hline Percentage İncrease (\%) & $32,5 \pm 32,7$ & $60,4 \pm 34,9$ & $0,000 \mathrm{~m}$ \\
\hline In-Group Change (p) & $0,000 w$ & $0,000 w$ & \\
\hline
\end{tabular}

${ }^{\mathrm{m}}$ Mann-whitney u test $/{ }^{\mathrm{w}}$ Wilcoxon test

Between the fetal heartbeat positive and negative groups, the serum $\beta$-hCG value at first admission did show no statistically significant difference (Pख0.05). The control $\beta$-hCG values obtained after 48 hours in both groups showed a significant increase $(P<0.05)$ compared to the first admission. In the group with 
fetal heartbeat positive, the increase rate between the two $\beta$-hCG values taken at 48 hour intervals was found to be significantly higher than the group without fetal heartbeat (P冈0.05).

The Figure- 1 shows the predictive power graph of serum $\beta-h C G$ increase rates taken at 48 hour intervals in the likelihood of fetal heartbeat. According to the chart, the probability of fetal heartbeat was $70 \%$ while the rate of increase in $\beta$-hCG value was $31 \%$, and the probability of fetal heartbeat was $80 \%, 90 \%, 95 \%$ and $100 \%$ while the rate of increase in $\beta$-hCG value was $49 \%, 73 \%, 97 \%$, and $181 \%$, respectively. Significant efficacy $(p=0.000)$ [0.748 $(0.621-0.874)$ of $\beta$-hCG increase was observed in predicting the groups with and without fetal heartbeat (Figure-2: ROC curve).

Table 5 : Comparison of serum Progesterone values between FHR +/- groups

\begin{tabular}{ccccc}
\hline \hline & Early Pregnancy Loss (FHB: - ) & & Detected Viability (FHB: + ) & $\mathrm{p}$ \\
\cline { 2 - 2 } & Mean \pm S.d. & Mean \pm S.d. & $17,1 \pm 8,1$ & $\mathbf{0 , 0 0 0 ~} \mathrm{m}$ \\
\hline Progesteron & $9,9 \pm 6,5$ & &
\end{tabular}

${ }^{\mathrm{m}}$ Mann-whitney u test /

When the initial serum progesterone values of the groups with and without fetal heartbeat were compared, a statistically significant difference was found between the groups (P:0.000). It was significantly higher in the FHR positive group compared to the negative group (Pख0.05). (Table-5). Significant efficacy $(p=0.000)$ [0.796 $(0.685-0.907)$ of the progesterone value was observed in predicting the group with and without fetal heart beat. (Figure-3: ROC curve)

When the serum progesterone value was $5.9 \mathrm{ng} / \mathrm{ml}$, the fetal heart rate was $49 \%$, and when the serum progesterone value was $10.5 \mathrm{ng} / \mathrm{ml}$ or above, the fetal heart rate was $69 \%, 12 \mathrm{ng} / \mathrm{ml}$ and above $75 \%, 13.4$ $\mathrm{ng} / \mathrm{ml}$ and above $80 \%, 18.0 \mathrm{ng} / \mathrm{ml}$ and above $90 \%, 21.7 \mathrm{ng} / \mathrm{ml}$ and above $95 \%, 29.3 \mathrm{ng} / \mathrm{ml}$ and above $99 \%$ and $37.5 \mathrm{ng} / \mathrm{ml}$ and above $100 \%$.

Table 6 : Comparison of TV-USG findings and measurements in FHR+/-groups

\begin{tabular}{|c|c|c|c|c|c|c|}
\hline & & \multicolumn{2}{|c|}{ Early Pregnancy Loss (FHB: -) } & \multicolumn{2}{|c|}{ Detected Viability (FHB: +) } & \multirow{2}{*}{$\mathrm{P}$} \\
\hline & & Mean \pm S.d./n- $\%$ & Median & Mean \pm S.d. $/ \mathrm{n}-\%$ & Median & \\
\hline \multirow{3}{*}{$\begin{array}{l}\text { Ultrasonography } \\
\text { (USG) }\end{array}$} & Only mGSD & 19 & $86.4 \%$ & 62 & $79.5 \%$ & \multirow{3}{*}{$0,468^{x^{2}}$} \\
\hline & $\begin{array}{l}\text { detected (No } \\
\text { CRL) }\end{array}$ & & & & & \\
\hline & CRL detected & 3 & $13.6 \%$ & 16 & $20.5 \%$ & \\
\hline \multicolumn{2}{|c|}{ USG measurement $(\mathrm{mm})$} & & & & & \\
\hline mGSD & & $10,8 \pm 4,7$ & 10,0 & $11,8 \pm 4,9$ & 12,0 & $0,419^{m}$ \\
\hline CRL & & $3,7 \pm 2,1$ & 3,2 & $3,4 \pm 1,2$ & 3,3 & $0,955^{\mathrm{m}}$ \\
\hline
\end{tabular}

${ }^{\mathrm{m}}$ Mann-whitney u test $/{ }^{\mathrm{x}^{2}}$ Ki-kare test

There was no statistically significant difference in ultrasound findings between the two groups. 


\section{Discussion:}

Transvaginal ultrasonography has always been a classical method to predict pregnancy outcomes in cases where viability is suspected in patients with early pregnancy, but many publications have recently studied $\beta$-hCG and progesterone values, which are serum biochemistry markers. Using these parameters, 336 pregnant women were followed up in our study, in which we investigated whether they were related to the determination of early pregnancy outcome. The results of our study, which was completed with 100 pregnant women, were compared with the literature.

Advanced maternal age is known to be associated with the risk of fetal anomalies, early pregnancy loss and increased abortion rates $(6,7)$. Some studies show that maternal age is an important parameter for predicting fetal viability (2). Our findings regarding maternal age are not consistent with the literature. Although the mean maternal age was higher in the group diagnosed with early pregnancy loss compared to the group with fetal viability, this difference was not found to be statistically significant. The reason for this was that the mean age was close to each other and the mean age of the group with early pregnancy loss failed to meet the advanced maternal age criterion ( $>35$ years) mentioned in the literature.

As far as we know, this study is the first to include the evaluation of gravida and parity numbers in prediction of early pregnancy. The literature includes only a comparison of abortion rates and it is known that repeated abortions increase the risk of early pregnancy loss $(6,7)$. In the previous pregnancy anamnesis, no statistically significant results were obtained when the numbers of gravida, parity and optional curettages were evaluated according to the results of early pregnancy. Although the number of abortus had a 2 times more average in the group without heartbeat compared to the group with heartbeat, the difference between these two groups was not found to be statistically significant contrary to the literature. Although no significant results were obtained, we contributed to the literature by evaluating the previous pregnancy anamnesis in early pregnancy loss, except for the history of abortus.

In the literature, the probability of intrauterine cystic structure belonging to the gestational sac is $99.5 \%$ and the probability of false sac is $0.5 \%$ (8). In ectopic pregnancies, the rate of false sac was reported to be $10 \%(9,10)$. In our study, 2 out of 100 patients were diagnosed with ectopic pregnancy. Considering that $81 \%$ of 100 patients had a gestational sac, the rate of false sac in patients with an observed gestational sac was $2.46 \%$. This is highly above the $0.5 \%$ rate stated in the literature. Considering that only $10 \%$ of ectopic pregnancies have a false sac, it is interesting that such a high rate was found only in a study on intrauterine pregnancies. This led us to conclude that clinicians should be more careful in ultrasonography examination and understand the distinction between double decidual ring appearance and actual gestational sac.

This study includes a race question in a society against racism since it is thought that abort rates may be high due to the high number of Syrian asylum seekers in our country, poor living conditions and war stress. Although high rates of early pregnancy loss have been reported in African-Americans compared to other populations in the literature $(6,7)$, no literature data was found on races and or studies conducted in the Middle East. No statistically significant results were obtained in the comparison between 
nationalities. The limited number of foreign patients participating in the study was considered as the reason for this.

Oh et al. (11) found that mGSD measured in pregnancies $<37$ days and below according to LMP had no predictive value for fetal outcome later in pregnancy. In another study, it was emphasized that there was no predictive value of gestational age before 37 days according to LMP, and a definitely unsuccessful pregnancy was given as any pregnancy with no observed gestational sac on the day 46 (12). Fritz et al. (13) found that gestational age and hCG doubling time were correlated and therefore, gestational age was prognostic for fetal outcome. Napolitano et al. (14) stated that $30 \%$ of women had an uncertain or unreliable menstrual date and therefore the gestational age could not be determined correctly. In our study, a statistically significant efficacy of gestational age was observed between the groups with and without fetal heartbeat according to the last menstrual period. This was found to be consistent with the general literature $(11,13)$. However, the information that the predictive value of gestational age was not significant before 37 days in the literature contradicted the occurrence of a heartbeat in all 7 women $(100 \%)$ diagnosed with pregnancy before 35 days in our study. Again, the information that the pregnancies detected after the day 46 mentioned in the literature were unsuccessful contradicted with the incidence of fetal heartbeat in $18(60 \%)$ of the 30 women diagnosed with pregnancy after the 46th day. However, the fact that $15 \%$ of the patients did not know their last menstrual periods and $11 \%$ were incorrect makes this result contradictory, and Napolitano et al. confirmed the argument that the last menstrual periods of $30 \%$ of the women was unreliable and concluded that the gestational age should be determined ultrasonographically or biochemically by experienced physicians.

Early pregnancy loss is known to be common in patients with symptoms $(1,6)$. Although our study includes patients with a higher rate of symptoms in the group without fetal heartbeat (in the FHR negative group: $31.8 \%$ - positive group: $17.4 \%$ ), this information was not found to be statistically significant. This was found to be inconsistent with the literature. The reason for this was construed as the fact that the study was conducted in a small population.

In the initial and 48-hour follow-up serum $\beta$-hCG controls, a statistically significant difference was found between the fetal heart rate positive group and the negative group. Claire et al. (1) found that serum $\beta$ hCG predicts viability with $100 \%$ sensitivity and $31 \%$ specificity when the doubling rate is $75 \%$ or more. Barnhart et al. (5) found the rate of determination of viability as $124 \%$ increase in 2 days. Bignardi et al. (12) found that the rate of determining viability with $\beta$-hCG doubled was $78 \%$ sensitive and $67 \%$ specific. As the main purpose of our study, the fact that $\mathrm{hCG}$ doubling rate provides prediction of viability is also consistent with many recent studies although the rates are different. What makes our study different is that the prediction of viability is mentioned by giving only certain ratios in the literature $(1,15)$, while in our study, a percentage is assigned to each patient in terms of viability at each increase rate of $\beta$-hCG (Figure-1). With this information, we believe that a clear rate will be provided to the patients during early pregnancy and that both the anxiety of the patients due to uncertainty will be reduced and their own anxiety will be reduced by physicians providing clear information and ratio to the patients. In addition to creating an early pregnancy prediction modality, our study examined the increase rates of $\beta$-hCG in the 
ROC curve and determined high sensitivity cut-off values and confirmed that the $\beta$-hCG increase rate in recent literature could be used to predict early pregnancy outcomes. In our study, live pregnancy was achieved even with a low rate of $\beta$-hCG increase of $31 \%$. Therefore, physicians should not diagnose pregnancy loss early, and early interventions should be avoided. The cut-off value determined in the $95 \%$ confidence interval was achieved with a minimum 97\% increase in hCG at the hour 48 . A $100 \%$ heart rate positivity was achieved with an increase of $181 \%$.

Claire et al. (1) found 100\% pregnancy failure where the progesterone value was $6.2 \mathrm{ng} / \mathrm{ml}$ or less. In a recent French guideline, an abnormal IUP or ectopic pregnancy was found to be associated with an initial serum progesterone value of $3.2 \mathrm{ng} / \mathrm{ml}$ or less (3). In our study, a significant statistical result was obtained when the initial progesterone values of the fetal heartbeat and non-fetal heartbeat groups were compared. This was found to be consistent with the general literature $(1,3)$. What makes our study different is that while the prediction of viability is mentioned in the literature only by giving certain ratios $(12,16)$, in our study, a percentage can be assigned to patients in terms of viability at each value of the initial serum progesterone (Figure-4). Thiis information makes it possible to give a clear rate to the patients during early pregnancy, and reducing the anxiety of families caused by waiting for weeks. Furthermore, our study examined initial serum progesterone on the ROC curve and determined high sensitivity cut-off values and confirmed that serum progesterone in the literature could be used to predict early pregnancy outcomes. A successful pregnancy was achieved even at an initial progesterone value of $3.71 \mathrm{ng} / \mathrm{ml}$. Therefore, the physicians are recommended to avoid early interventions. Besides, the cut-off value determined in the $95 \%$ confidence interval was provided at $21.7 \mathrm{ng} / \mathrm{ml}$ and above. A $100 \%$ heart rate positivity was achieved with values of $37.5 \mathrm{ng} / \mathrm{ml}$ and above. The reason why we found slightly higher cut-off values compared to the general literature is the low number of patients, which is also the limitation of our study .

The $22 \%$ early pregnancy loss rate obtained as a result of our study is higher than the EEC rate of 10$15 \%$ in the literature, but also lower than the EEC rate of $31 \%$ after implantation $(1,6,7)$. Although our population was a heterogeneous group of healthy and symptomatic patients, the presence of symptomatic patients at a higher rate than the rate in the community was considered as the likely reason for the higher rate of our EEC rates than the literature statistics. The reason why our EEC rate was lower than the EEC rate observed after implantation was considered as the fact that patients with EEC were not evaluated without creating an intrauterine finding due to the inclusion of IUPs only with TV-USG in our population. Although the incidence of embryos was higher in the FHR positive group compared to the negative group, the incidence of gestational sacs or embryos between the FHR +/- groups was not statistically significant. This result is inconsistent with the literature because the literature states that the presence of embryos is more valuable than other ultrasound findings (yolk sac, GS) and it is among the good prognostic factors $(2,3)$. Pexster et al. (17) found variations up to $20 \%$ between the operators in $\mathrm{CRL}$ and mGSD measurement accuracy. It is possible to see many variations and cut-off values in the literature for diagnosis and definitions (18). Although our population is not as heterogeneous as the community and we do not study inter-operator variations, we think that ultrasound is a subjective 
diagnostic tool. This is perhaps another reason why our ultrasound findings were not statistically significant in predicting early pregnancy outcomes.

\section{Conclusion:}

In our study, the doubling ratio of serum hCG and the initial single value of serum progesterone were found to be highly significant in predicting early pregnancy outcomes in accordance with the literature. The difference of our study from the literature is that the patients are not given only a cut-off but a percentage in terms of viability at every increase rate of $\beta$-hCG and every value of serum progesterone. This prediction modality made a significant contribution to the literature. The probability of FHR was $70 \%$ while the $\beta$-hCG increase rate was $31 \%$, and the probability of FHR was $80 \%, 90 \%, 95 \%$ and $100 \%$ while the $\beta$-hCG increase rate value was $49 \%, 73 \%, 97 \%$, and $181 \%$, respectively. When the progesterone value was $5.9 \mathrm{ng} / \mathrm{ml}$, the probability of FHR was $49 \%$, and $69 \%$ at $10.5 \mathrm{ng} / \mathrm{ml}, 80 \%$ at $13.4 \mathrm{ng} / \mathrm{ml}, 90 \%$ at 18.0 $\mathrm{ng} / \mathrm{ml}, 95 \%$ at $21.7 \mathrm{ng} / \mathrm{ml}, 99 \%$ at $29.3 \mathrm{ng} / \mathrm{ml}$, and $100 \%$ at $37.5 \mathrm{ng} / \mathrm{ml}$ and above. This information confirms that the doubling rate of serum $\beta-h C G$ at the hour 48 and serum progesterone can be used to predict early pregnancy outcomes, and an estimation modality was created.

This study did not evaluate female anxiety or female satisfaction. However, there are observational findings that it provides psychological benefits. The limitation of our study was the small number of patients. Another limitation was that due to patients' previous pregnancy experiences and sociodemographic factors, patients who we predicted would be negative for FHR but could not demonstrate this ultrasonographically acknowledge this. In this study, prediction scores for early pregnancy outcomes incorporating clinical signs, biological markers, or ultrasound findings in early pregnancy outcomes were developed, but a few studies are not sufficient to set aside TV-USG. Furthermore, the superiority of serum $\beta$-hCG and serum progesterone levels to each other was not evaluated in our study. By disseminating similar studies on this subject and increasing patient participation, early pregnancy outcomes can be determined earlier and clearer rates can be found. Besides, TV-USG is an expensive examination tool for western countries, even if not for our country. Reducing subjectivity due to operators such as ultrasound and obtaining early results with diagnostic tools that do not require human interpretation can help reducing legal problems, families' anxiety, hospital admissions as well as health costs.

\section{Abbreviations}

IUP : intrauterine pregnancy

CRL : Crown-rump Length

TV-USG : Transvaginal Ultrasonography

FHR : Fetal heart rate

FHR+ positive : Viable

Page $11 / 16$ 
FHR- negative : Non-viable, early pregnancy loss

PUL : Pregnancy of Unknown Locations

PUV : Pregnancy of Uncertain Viability

mGSD : mean of three orthogonal measurements Gestastional Sac Diameter

ROC : Receiver Operating Characteristic

LMP : Last menstrual period

\section{Declarations}

\section{Ethics approval and consent to participate}

Our study was carried out in the Obstetrics and Gynecology Clinic of Istanbul Okmeydani City Hospital on a single-center "Prospective observational" basis between March 2018 and December 2018. The study was commenced after obtaining the approval of the Ethics Committee for Clinical Research of Istanbul Okmeydani City Hospital under the protocol number 841 on 13/03/2018. The participants were informed verbally and in writing, and their written consents were obtained. The original of the document is available in the hospital archive. All methods were carried out in accordance with relevant guidelines and regulations.

\section{Consent for publication}

We obtained approval from the Istanbul Okmeydani Prof.Dr. Cemil Tasci City Hospital Ethics Committee for the study. In accordance with the "Patient Rights Regulation" (Date: 01.08.1998; Issue: 23420), which was prepared in accordance with international standards, verbal and wet signed written consent was obtained from the patient under the title of "Consent to publish"' in order to publish patient information and support public health. A wer signed copy of the document is attached.

\section{Availability of data and material}

For this Research according to the "Patient Rights Regulation" 01.08.1998 dated and numbered 23420, patient information is allowed to be published with the permission of the patient. Ethics committee approval was obtained and documents regarding permission to publish were obtained from the patients and kept in the archive of Prof Dr Cemil Tascioglu City Hospital. Patient data and materials used in this research study are available in our hospital's electronic data warehouse and archive. The following links are available.

https://cemiltascioglush.saglik.gov.tr/

https://cemiltascioglush.istanbulsaglik.gov.tr/hastaportal/\#/?p=results 


\section{Competing interests}

We declare that there is no conflict of interest between the authors. All authors are in agreement with the design, progress, evaluation and results of the study. Statement of Authorship form and Approval of The Responsible Authors form is attached.

\section{Funding}

No resources or grants contributed to the completion of this study. The document is attached.

\section{Authors' contributions}

From the authors; MY (Corresponding author), contributed to the conception and design of the study, acquisition of data, analysis and interpretation of data, drafting the article and revising it critically for important intellectual content, and finally final approval of the version to be submitted. ENC; contributed by acquisition of data, analysis and interpretation of data. GD and OS helped us to the conception and design of the study. YO; contributed to the preparation and translation of the article. VM provided the final approval of the version to be presented. All authors have read and approved the manuscript. Approval of The Authors form is attached.

\section{Acknowledgements}

No resources or grants contributed to the completion of this study.

\section{References}

1. Claire P, Yolaine J, Estelle B, Bruno L, Claude B, Vincent L, Maela LL. Serial hCG and progesteron elevels to predict early pregnancy outcomes in pregnancies of uncertain viability: A prospective study. EJOGRB 220 (2018) 100-105, doi: https://doi.org/10.1016/J.EJOGRB.2017.11.020.

2. Wilcox AJ, Weinberg CR, O'Connor JF, Baird DD, Schlatterer JP, Canfield RE, et al. Incidence of early loss of pregnancy. New Engl J Med 1988;319:189-94, doi: http://dx.doi.org/10.1056/NEJM198807283190401.

3. Lavoué V, Huchon C, Deffieux X, Voltzenlogel M-C, Vandenbroucke L, Levêque J. French guidelines for diagnostic criteria for non viable pregnancy early in the first trimester and for management of threatened miscarriage and non viable pregnancy (except uterine evacuation). J Gynecol Obstet Biol Reprod (Paris) 2014;43:776-93, doi:http://dx.doi.org/10.1016/j.jgyn.2014.09.012.

4. Petrou S, Trinder J, Brocklehurst P, Smith L. Economic evaluation of alternative management methods of first-trimester miscarriage based on results from the MIST trial. BJOG, 2006; 113: 87989, doi:http://dx.doi.org/10.1111/j.1471-0528.2006.00998.x.

5. Barnhart KT, Sammel MD, Rinaudo PF, Zhou L, Hummel AC, Guo W. Symptomatic patients with an early viable intrauterine pregnancy: HCG curves redefined. Obstet Gynecol. 2004 Jul;104(1):50-5. doi: 10.1097/01.AOG.0000128174.48843.12. 
6. The American Journal of Obstetricians and Gynecologists. Early Pregnancy Loss. Practice Bulletin 2015; 150.

7. The Ultrasound Advisory Group. Addendum to GTG No 25 (Oct 2006): The Management of Early Pregnancy Loss. 2011.

8. Doubilet PM, Benson CB, Bourne T, Blaivas M, Barnhart KT, Benacerraf BR, et al. Diagnostic criteria for nonviable pregnancy early in the first trimester. Society of Radiologists in Ultrasound Multispecialty Panel on Early First Trimester Diagnosis of Miscarriage and Exclusion of a Viable Intrauterine Pregnancy. N Engl J Med 2013;369:1443-51.

9. Rodgers SK, Chnag C, DeBardeleben JT, Horrow MM. Normal and Abnormal US Findings in Early First-Trimester Pregnancy: Review of the Society of Radiologists in Ultrasound 2012 Consensus Panel Recommendations. Radio Graphics 2015; 35: 2135-48.

10. Barnhart KT. Clinical practice. Ectopic pregnancy. N Engl J Med. 2009;361:379-87. doi: 10.1056/NEJMcp0810384.

11. Oh JS, Wright G, Coulam CB. Gestational sac diameter in very early pregnancy as a predictor of fetal outcome. Ultrasound Obstet Gynecol. 2002 Sep;20(3):267-9. doi: 10.1046/j.14690705.2002.00774.x.

12. Bignardi T, Condous G, Kirk E, Van Calster B, Van Huffel S, Timmerman D, Bourne T. Viability of intrauterine pregnancy in women with pregnancy of unknown location: prediction using human chorionic gonadotropin ratio vs. progesterone. Ultrasound Obstet Gynecol. 2010 Jun;35(6):656-61. doi: 10.1002/uog.7669.

13. Fritz MA, Guo SM. Doubling time of human chorionic gonadotropin (hCG) in early normal pregnancy: relationship to hCG concentration and gestational age. Fert Ster. 1987 Apr;47(4):584-9.

14. Napolitano R, Dhami J, Ohuma EO, loannou C, Conde-Agudelo A, Kennedy SH, et al. Pregnancy dating by fetal crown-rump length: a systematic review of charts. BJOG 2014; 121:556-665.

DOI:10.1111/1471-0528.12478.

15. Bree RL, Edwards M, Böhm-Vélez M, Beyler S, Roberts J, Mendelson EB. Transvaginal sonography in the evaluation of normal early pregnancy: correlation with HCG level. AJR Am J Roentgenol. 1989 Jul;153(1):75-9.

16. Verhaegen J, Gallos ID, van Mello NM, Abdel-Aziz M, Takwoingi Y, Harb H, Deeks JJ, Mol BW, Coomarasamy A. Accuracy of single progesterone test to predict early pregnancy outcome in women with pain or bleeding: meta-analysis of cohort studies. BMJ. 2012 Sep 27;345:e6077. doi: 10.1136/bmj.e6077.

17. Pexsters A, Luts J, Van Schoubroeck D, Bottomley C, Van Calster B, Van Huffel S, et al. Clinical implications of intra and interobserver reproducibility of transvaginal sonographic measurement of gestational sac and crown-rump length at 6-9 weeks' gestation. Ultrasound Obstet Gynecol. 2011 Nov;38(5):510-5. doi: 10.1002/uog.8884.

18. Abdallah Y, Daemen A, Kirk E, Pexsters A, Naji O, Stalder C, et al. Limitations of current definitions of miscarriage using mean gestational sac diameter and crown-rump length measurements: a multi 
center observational study. Ultrasound Obstet Gynecol. 2011 Nov;38(5):497-502. doi:

10.1002/uog.10109.

Figures

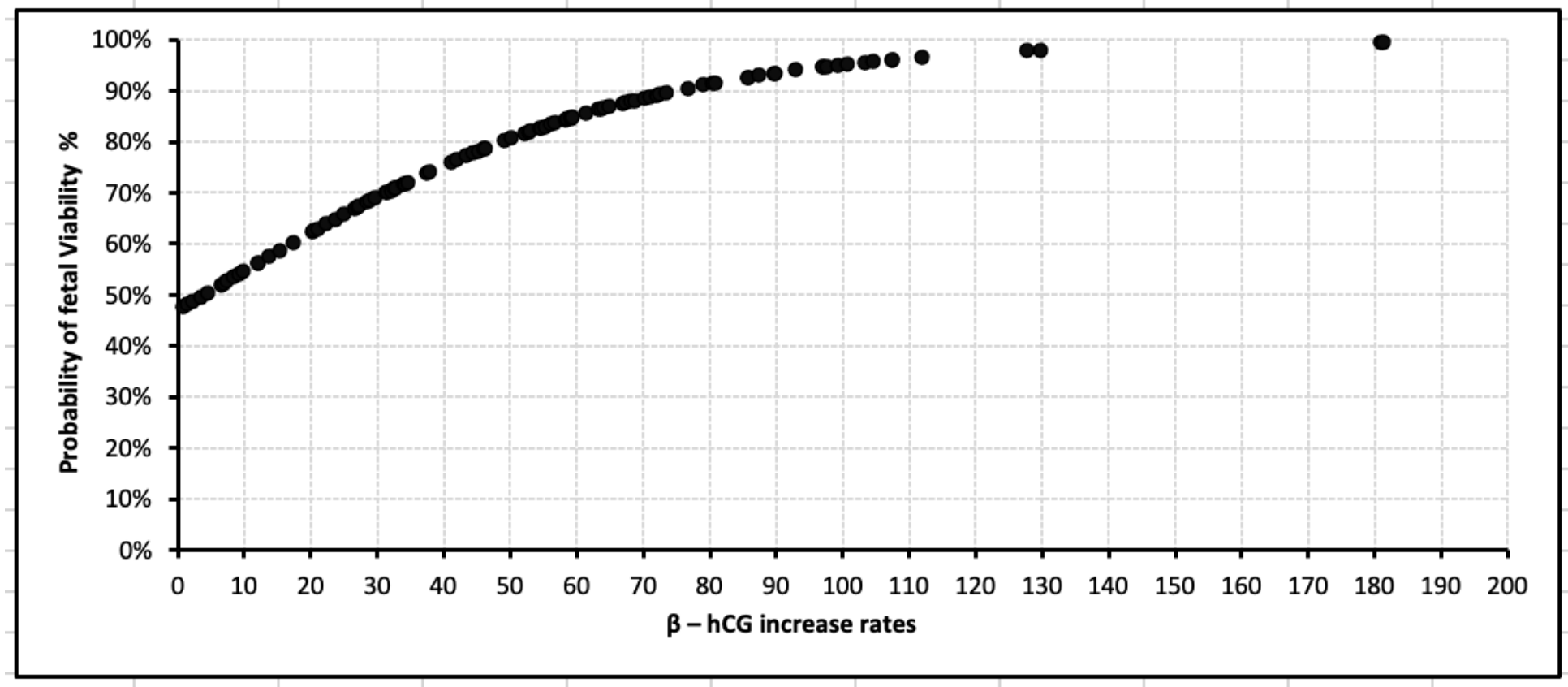

Figure 1

Chart of the likelihood of fetal heartbeat with serum $\beta$-hCG increase rates

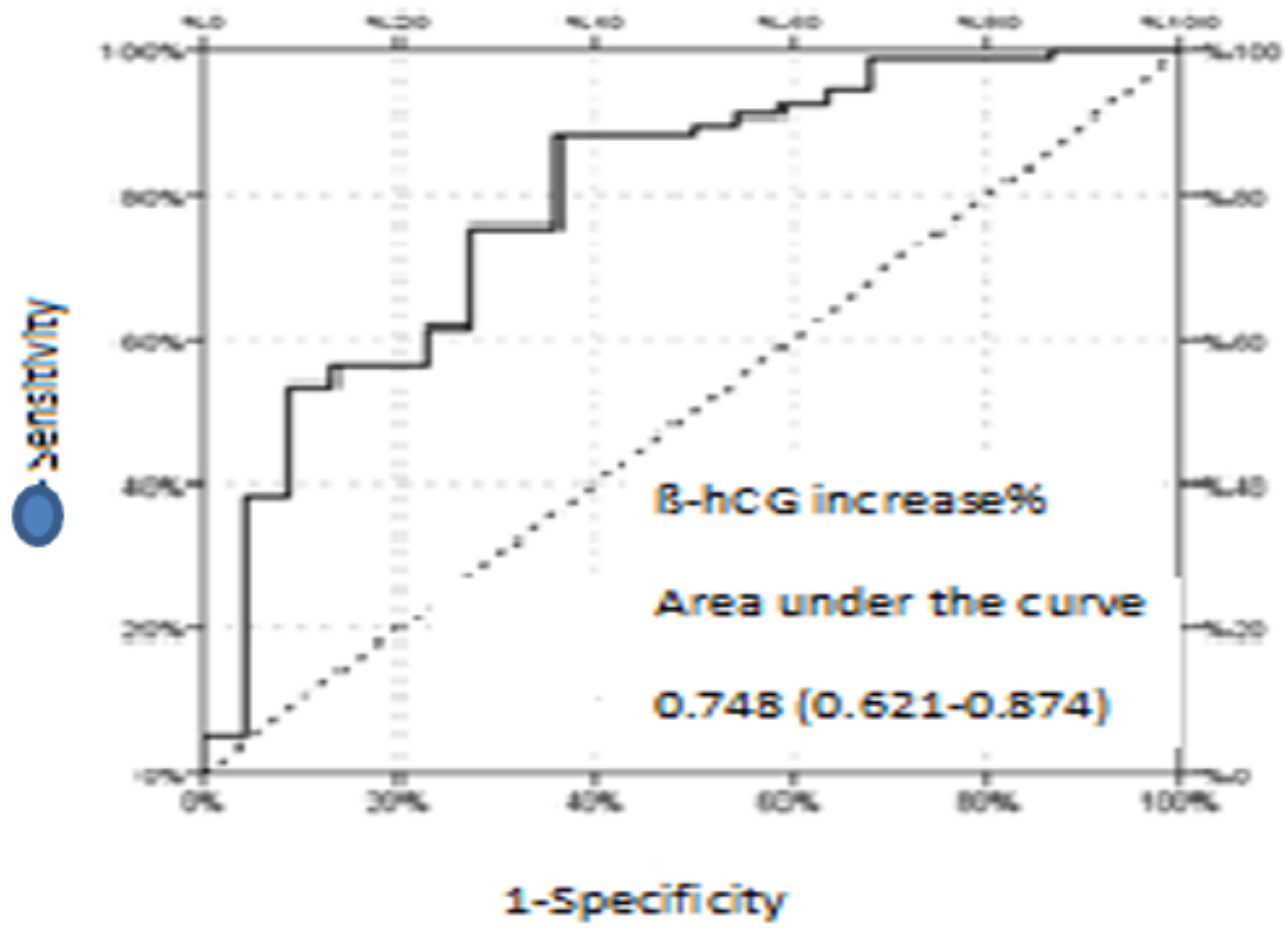

Figure 2 
$\mathrm{ROC}$ analysis of $\beta$-hCG increase percentage in $\mathrm{FHR}+/$ - group prediction

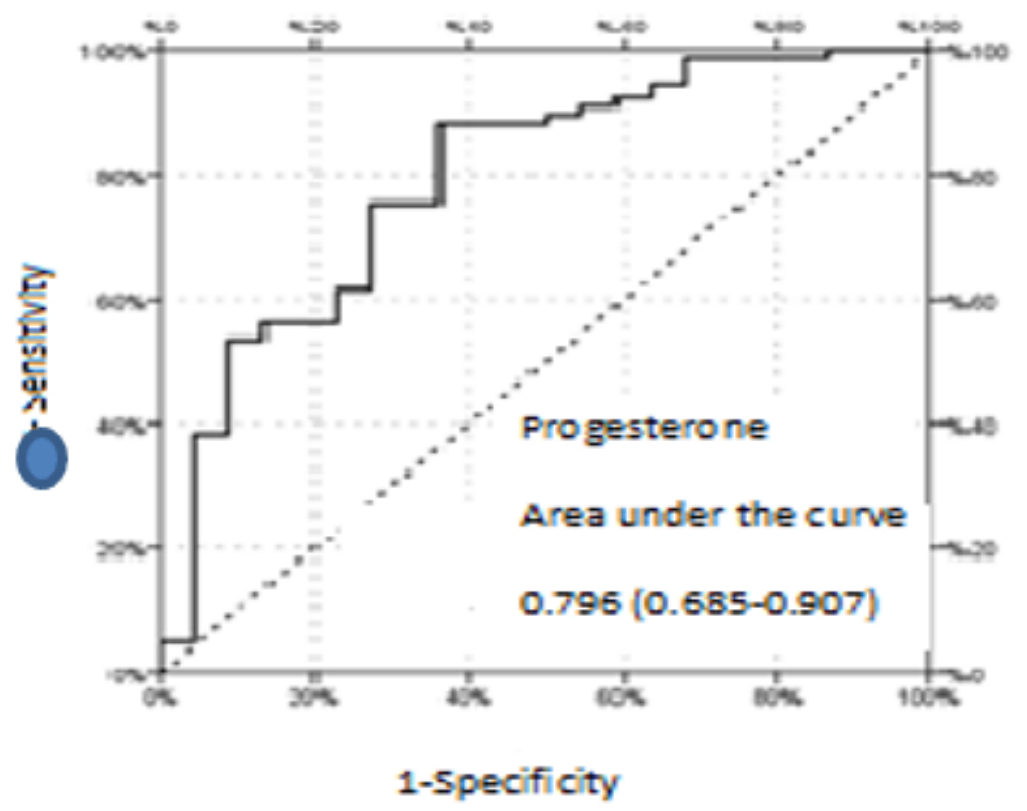

Figure 3

Serum Progesterone level ROC analysis in FHR +/- group prediction

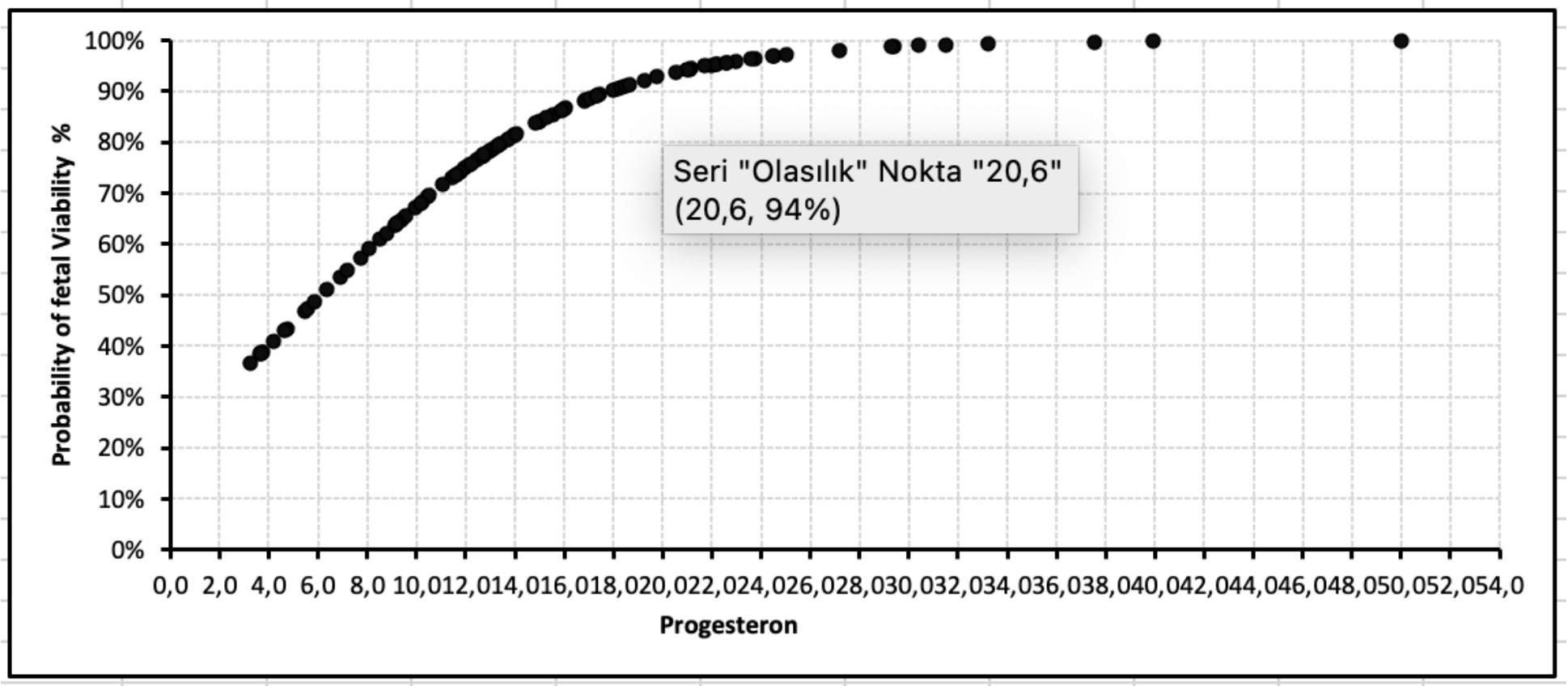

Figure 4

Chart of initial serum Progesterone value and probability of fetal heart rate 